\title{
Effect of clinical parameters on the control of myoelectric robotic prosthetic hands
}

\author{
Manfredo Atzori, PhD; ${ }^{1 *}$ Arjan Gijsberts, PhD; ${ }^{2}$ Claudio Castellini, PhD; ${ }^{3}$ Barbara Caputo, PhD; ${ }^{2}$ Anne-Gabrielle \\ Mittaz Hager, PhD; ${ }^{4}$ Simone Elsig; ${ }^{4}$ Giorgio Giatsidis, MD; ${ }^{5}$ Franco Bassetto, MD; ${ }^{5}$ Henning Müller, $\mathbf{P h D}^{\mathbf{1}}$ \\ ${ }^{1}$ Information Systems Institute at the University of Applied Sciences Western Switzerland (HES-SO Valais), Sierre, \\ Switzerland; ${ }^{2}$ Department of Computer, Control, and Management Engineering, University of Rome, Roma, Italy; \\ ${ }^{3}$ Robotics and Mechatronics Center of the DLR-German Aerospace Center, Oberpfaffenhofen, Germany; ${ }^{4}$ School of \\ Health Sciences, Physiotherapy at the University of Applied Sciences Western Switzerland (HES-SO Valais), Leukerbad, \\ Switzerland; ${ }^{5}$ Clinic of Plastic Surgery, Padova University Hospital, Padova, Italy
}

\begin{abstract}
Improving the functionality of prosthetic hands with noninvasive techniques is still a challenge. Surface electromyography (sEMG) currently gives limited control capabilities; however, the application of machine learning to the analysis of sEMG signals is promising and has recently been applied in practice, but many questions still remain. In this study, we recorded the sEMG activity of the forearm of 11 male subjects with transradial amputation who were mentally performing 40 hand and wrist movements. The classification performance and the number of independent movements (defined as the subset of movements that could be distinguished with $>90 \%$ accuracy) were studied in relationship to clinical parameters related to the amputation. The analysis showed that classification accuracy and the number of independent movements increased significantly with phantom limb sensation intensity, remaining forearm percentage, and time since amputation. The classification results suggest the possibility of naturally controlling up to 11 movements of a robotic prosthetic hand with almost no training. Knowledge of the relationship between classification accuracy and clinical parameters adds new information regarding the nature of phantom limb pain as well as other clinical parameters, and it can lay the foundations for future "functional amputation" procedures in surgery.
\end{abstract}

Key words: myoelectric prosthesis, phantom limb pain, phantom limb sensation, prosthesis, prosthetic hand, residual limb, residual limb length, robotic prosthesis, sEMG, transradial amputation.

\section{INTRODUCTION}

Most upper-limb amputations are transradial [1]. In order to recover some functional capabilities, subjects with transradial amputation can either use a kinematic prosthesis, in which the motion of the shoulders controls the opening and closing of a gripper, or rely on a myoelectric prosthesis, in which the amplitude of the electrical signals emitted by the remnant muscles in the residual limb is used to control a prosthetic hand. In most cases, the movements that the subjects can perform are limited to opening and closing. The range and complexity of movements can be increased by employing a sequential control strategy. Controlling such prostheses requires a high

\footnotetext{
Abbreviations: DASH $=$ Disabilities of the Arm, Shoulder and Hand; $k$-nn $=k$-nearest neighbors; LSSVM = least squares support vector machines; RMS = root mean square; $\mathrm{sEMG} \mathrm{=}$ surface electromyography; SVM = support vector machines; $\mathrm{WL}=$ waveform length.

*Address all correspondence to Dr. Manfredo Atzori, University of Applied Sciences Western Switzerland, Sierre (HES-SO Valais), Rue du Technopole 3, 3960 Sierre, Switzerland; +41-27-606-9023; fax: +41-27-606-9000.

Email: manfredo.atzori@hevs.ch

http://dx.doi.org/10.1682/JRRD.2014.09.0218
} 
level of skill, and the training is long and complicated. These control problems contribute to the scarce capabilities and limited acceptance of surface electromyography (sEMG) prostheses [1]. Recently, a clinically deployed pattern-recognition system was introduced commercially (http://www.coaptengineering.com/). The system relies on improvements similar to the ones that have been described in the scientific literature [2-12]. Most of these methods rely on the use of several electrodes to record the myoelectric signals from the residual limb and pattern recognition algorithms to classify the movement that the subject aims to perform.

However, there are still several questions in the field that remain unsolved. First, most of the scientific investigations do not translate into a general description of the problem. There is no clear understanding of all the factors involved and of how several factors (such as clinical parameters, surgical procedures, electrode positioning, signal features, and classification method) interact. Thus, given a patient with a specific clinical situation, it is not clear which approach described in literature would lead to the best performance. The acquisition protocols described in the literature are heterogeneous and often not comparable; the data are usually not publicly available, and the clinical parameters of the subjects are considered in only a few studies. Thus, the results of the analyses (as well as the relationship with clinical parameters) usually cannot be compared. Second, the data sets are usually small, both considering the number of subjects [13] and the number of movements [5]. Third, despite a few articles addressing single problems, it is so far unknown how clinical parameters related to the amputation (e.g., remaining forearm percentage, phantom limb sensation, use of prostheses [7]) and physiological phenomena (e.g., cortical reorganization [14]) interact and affect the natural control capability of the prosthesis.

Excellent results have been obtained by targeted muscular reinnervation [9], but this technique is invasive. In a few noninvasive studies, the authors were able to obtain classification accuracies greater than 90 percent for specific subjects with transradial amputation [2,7], but average accuracies are usually below 80 to 90 percent for fewer than 20 movements [8]. Moreover, the studies sometimes present unbalanced classification results (i.e., with an unequal distribution of elements between classes) and include rest (which is usually repeated more frequently than other movements and classified with very high accuracy) in the analysis. Most standard learning algorithms expect balanced class distributions or equal misclassification costs. Therefore, they can fail to properly represent the characteristics of the data when dealing with imbalanced data sets [15] and obfuscate real performance in distinguishing between movements.

An important shortcoming of all of the mentioned aspects is that very few studies have evaluated the statistical relationships between classification accuracy and clinical variables of subjects with transradial amputation. One of the few relevant articles in this field describes differences in control accuracy based on the type of prosthesis regularly used [7]; however, to the best of our knowledge, there are no studies considering the effects of most of the clinical parameters (including remaining forearm percentage, phantom limb sensation intensity, or time since amputation).

The NinaPro project (Non-Invasive Adaptive Hand Prosthetics; http://ninaweb.hevs.ch/) aims to help the scientific community overcome the problems with sEMGcontrolled hand prosthetics through the creation of a benchmark database to evaluate machine learning algorithms for hand prosthesis control [16].

In this study, we analyzed the relationship between clinical parameters related to the amputation and the classification accuracy of the sEMG signals. The movement classification accuracies were analyzed for 11 subjects with transradial amputation who were performing 40 hand and wrist movements (plus rest) using 9 state-of-the-art machine-learning classifiers and 5 feature groups extracted from the signals; moreover, for each subject, we identified subsets of movements that were classified with greater than 90 percent accuracy, which can lead to high-quality control of a dexterous robotic hand with sufficient flexibility.

The analysis of the clinical parameters in relation to the classification of sEMG signals revealed interesting significant relationships between the capability to recognize movements in subjects with amputation and remaining forearm percentage, phantom limb sensation, and time since amputation. To our knowledge, such relationships have not been previously described. Thus, they open the field to possible interpretations that could improve the knowledge of the clinical parameters and their exploitation. In particular, these results can increase the quality of life and the prognosis for subjects with transradial amputation because modern prostheses can be 
improved by being adapted to the clinical characteristics of the subjects.

\section{METHODS}

\section{Subjects}

Eleven subjects with transradial amputation participated in this study. The subjects completed a questionnaire that included generic parameters (age, sex, height, weight, and handedness) and a detailed assessment of the clinical parameters related to the amputation (phantom limb sensation and phantom limb pain). To facilitate the participants' understanding of the pain categories, we described the types of pain and sensation in the following ways: "phantom limb" was defined as the portion of the limb that was amputated or missing; "nonpainful phantom limb sensations" were defined as sensations in the missing (phantom) limb that were not painful (e.g., the feeling that the missing hand is wrapped in cotton); and "phantom limb pain" referred to painful sensations in the phantom limb. The questions included side, level, date, and reason for amputation; forearm circumference; intensity and type of phantom limb sensation; presence of phantom limb pain; suffering due to phantom limb pain; use of prostheses (cosmetic, kinematic, myoelectric; years of use and daily use); and Disabilities of the Arm, Shoulder and Hand (DASH) score [17]. The average intensity of the phantom limb sensation was assessed on a 6 -point numerical rating scale $(0=$ no sensation; $5=$ sensation as strong as it could be). As suggested by the Chronic Pain Grade [18], a 3 to 6 mo recall period was used to assess average phantom limb sensation and pain intensity.

The subjects with amputation were all males with an average age of $42.36 \pm 11.96$ yr. All subjects except one were originally right handed. The amputated forearm is the right one in seven cases, the left one in three cases, while one subject underwent bilateral forearm amputation. All subjects were amputated because of traumatic injury, except one that was amputated because of a tumor. The remaining forearm percentage of the subjects is represented in Figure 1.

The experiment was conducted according to the principles expressed in the Declaration of Helsinki, and it was approved by the Ethics Commission of the Canton of Valais (Switzerland). All participants signed an informed consent form. The study with the highest number of sub-

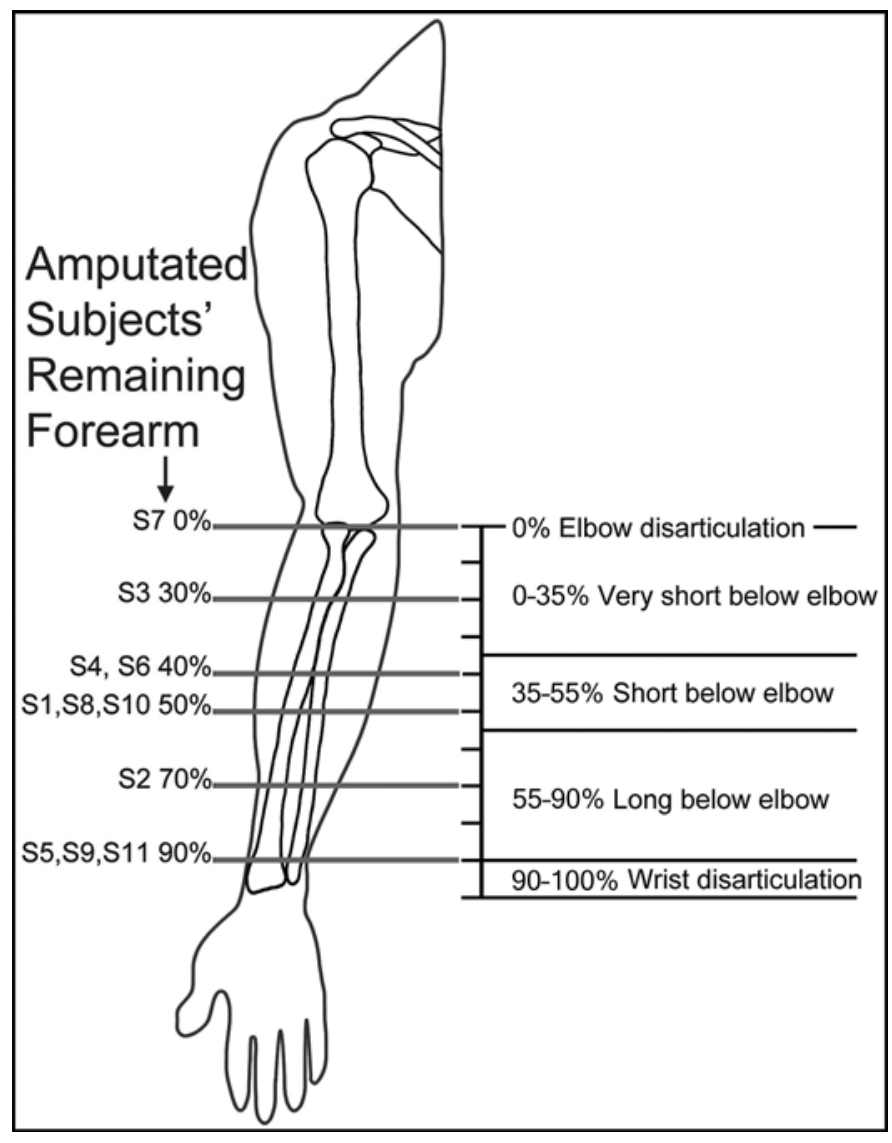

Figure 1.

Remaining forearm percentage of subjects with amputation. $\mathrm{S}=$ subject.

jects with amputation before this project included only six subjects [8]. The data recorded from the 11 subjects with transradial amputation are publicly available from the NinaPro database [19] (http://ninapro.hevs.ch).

\section{Acquisition Protocol}

The data are included in the third NinaPro database and were acquired according to the final version of the NinaPro acquisition protocol, which is thoroughly described elsewhere [19]. During the acquisition, the subjects were asked to mimic the movements shown on the screen of a laptop with the missing limb as naturally as possible (Figure 2) [1]. The experiment included 6 repetitions of 40 movements and 9 force patterns (Figure 3) [20]. The movements were selected from the most recent hand taxonomy and robotics literature $[4,13,21-$ 25 ] in order to allow performance of most of the activities needed in daily life. Each movement repetition lasted 


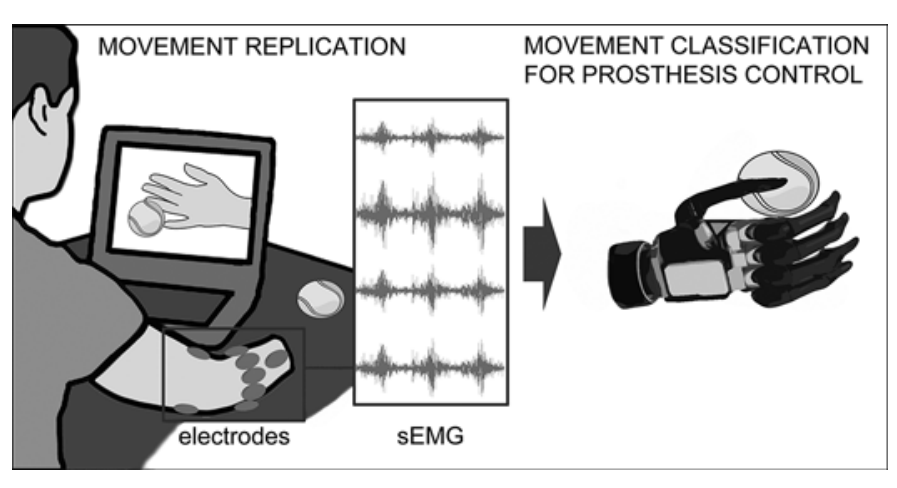

Figure 2.

Acquisition setup and acquisition procedure of the experiment. sEMG = surface electromyography.

$5 \mathrm{~s}$ and was followed by $3 \mathrm{~s}$ of rest (which is also considered in the analysis).

The electrodes were positioned as shown in Figure 2: eight electrodes were equally spaced around the forearm at the height of the radio-humeral joint, two electrodes were placed on the main activity spots of the flexor digitorum superficialis and the extensor digitorum superficialis muscles [16], and two electrodes were placed on the main activity spots of the biceps brachii and the triceps brachii muscles. The described locations were chosen in order to combine two positioning strategies widely used in the field (i.e., dense sampling [5-6,26] and precise anatomical positioning [27-28]). The positioning of electrodes on the upper arm is not common in the literature, but it can be helpful to improve the classification accuracy, in particular to include multimodal data in subjects with a low percentage of the forearm remaining. In two cases (subjects 6 and 7), the number of electrodes was reduced to 10 because of insufficient space. In one case (subject 7), the electrodes were placed on the border of the residual limb, with the aim of acquiring all the possible sEMG activity from the remnant muscles. This is obviously not optimal, but it was the only feasible approach for this particular subject.

The experiment was divided into one training session and three exercise sessions that addressed different types of movements, interrupted by rest time to avoid muscular fatigue. Three subjects asked to interrupt the experiment before its end because of fatigue or pain. In only one case was the number of movements less than the ideal number of movements considered in this study (38 movements) (Table 1).

\section{Data Analysis}

\section{Feature Extraction and Classification}

Our aim in selecting the algorithms we used was to offer a broad analysis of the relationships between clinical parameters and the natural control capabilities of robotic hand prostheses by people with transradial amputation. The classification procedure was relatively standard for the field; it followed the guidelines of Englehart and Hudgins [29] and consisted of preprocessing, windowing, feature extraction, and classification, performed as described in Atzori et al. [19].

The choice of window length trades the balance between prediction delays and classification accuracy [30]. Since, in this work, we were primarily interested in classification accuracy, we chose a relatively long window length of 400 ms. Furthermore, it is important to realize that window length is not identical to prediction delay, and previous studies in the NinaPro database have shown that the delay in predicting the correct movement for this window length is not necessarily much higher than with shorter window lengths [20].

Four movement repetitions were used to generate the training features $[1,3-4,6]$, while the remaining two were used to create the test set $[2,5]$. The classification was performed on the kinematics of the movements shown in Figure 3 [19], and it was balanced according to the number of movement repetitions through random undersampling. It has been demonstrated that the success of an sEMG pattern-recognition system strongly depends upon the choice of the signal features used to represent it [30]. Moreover, different signal features often have different computational requirements, and they can highlight different characteristics of the signal (also in relationship to clinical characteristics). Thus, we considered 5 signal features extracted from the signals and 9 classification methods, selected based on popularity, previous application to sEMG [20,29,31], and approach diversity. In several cases, the features can be referred to as feature sets rather than features, and they include the combination of all features as well. Root mean square (RMS) is probably the most commonly used feature representation for sEMG signals. RMS is easily implementable, and under ideal conditions, it has a quasi- or curvilinear relationship between its value and the force exerted by a muscle [27]. Waveform length (WL) measures the length of a given waveform, which is also a measure of the signal complexity. WL was proven to be a robust and efficient feature for analysis of sEMG [32], and it was already 


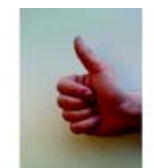

1. Thumb up

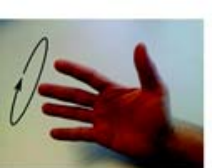

9. Wrist supination (axis: middle finger)

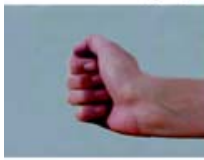

17. Wrist extension with closed hand

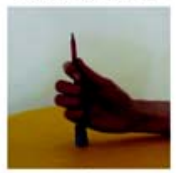

25. Stick grasp

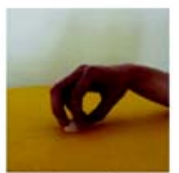

33. Quadpod grasp

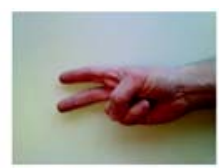

2. Extension of index and middle, flexion of the others

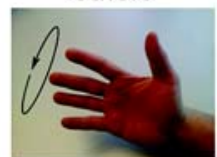

10. Wrist

pronation (axis: middle finger)

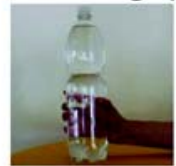

18. Large diameter grasp

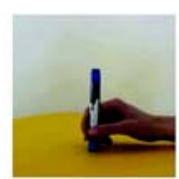

26. Writing tripod 27. Power sphere grasp

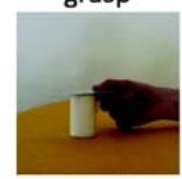

34. Lateral grasp

35. Parallel

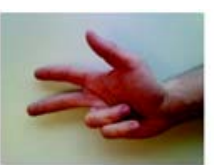

3. Flexion of ring and little finger, extension of the others

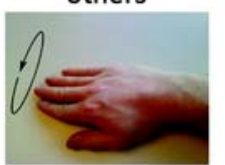

11. Wrist

supination (axis: little finger)

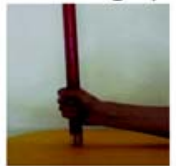

19. Small diameter grasp (power grip)
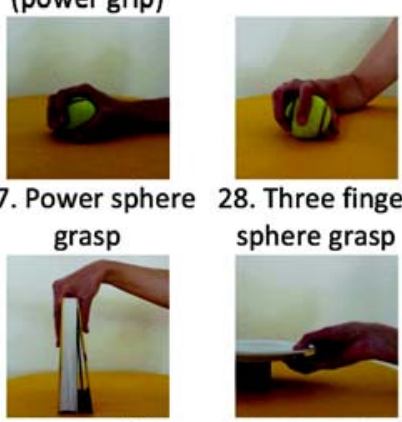
extension grasp
28. Three finger sphere grasp

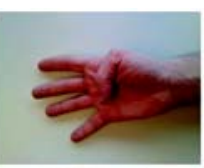

4. Thumb opposing base of little finger

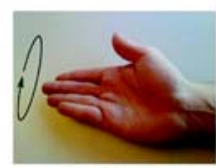

12. Wrist pronation (axis: little finger)

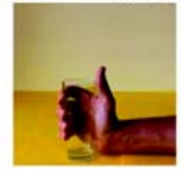

20. Fixed hook grasp

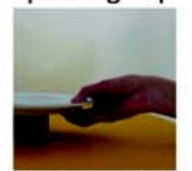

36. Extension type grasp

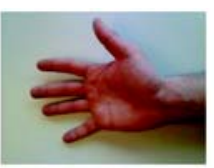

5. Abduction of all fingers

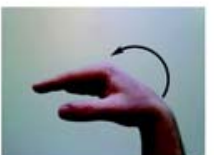

13. Wrist flexion

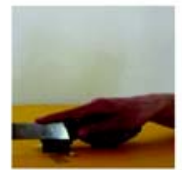

21. Index finger extension grasp

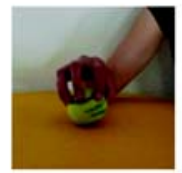

29. Precision sphere grasp

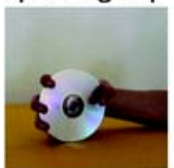

37. Power disk grasp

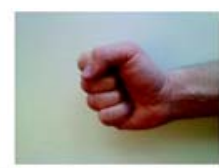

6. Fingers flexed
together in fist

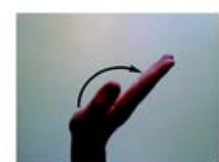

14. Wrist extension

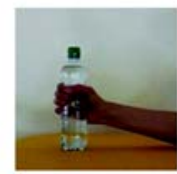

22. Medium wrap

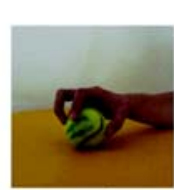

30. Tripod grasp

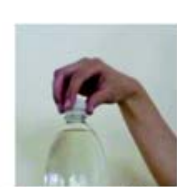

38. Open a bottle with a tripod grasp

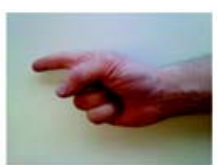

7. Pointing index

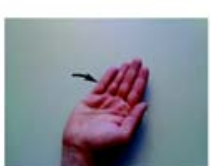

15. Wrist radial deviation

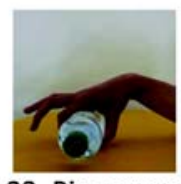

23. Ring grasp

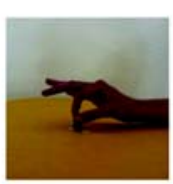

31. Prismatic pinch grasp

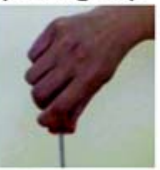

39. Turn a screw 40 . Cut something (grasp the screwdriver with a stick grasp) extended finger

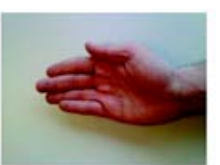

8. Adduction of

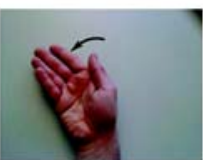

16. Wrist ulnar deviation

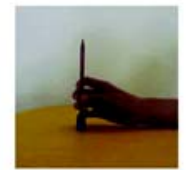

24. Prismatic four fingers grasp

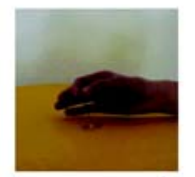

32. Tip pinch grasp

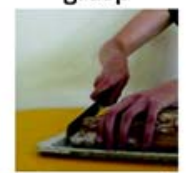

(grasp the knife with an index finger extension grasp)

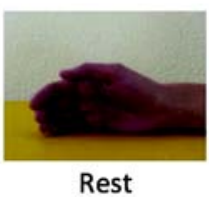

Figure 3.

List of analyzed movements.

applied in the NinaPro database [31]. sEMG histogram is the histogram of the time window given a predefined number of bins [33]. This feature has previously demonstrated excellent movement classification performance on sEMG [31,33]. The upper and lower thresholds of the standardized sEMG were set to three standard deviations. The total number of bins was 20 and the extremal ones were defined to capture the outliers, leading to the fol- lowing edges: $[-\infty,-3, \ldots,+3,+\infty]$. The marginal Discrete Wavelet Transform uses a wavelet basis function to decompose the signal at several resolution levels, leading to a frequency-time representation that is high-dimensional. In order to reduce the dimensionality of the feature, only the marginals of the decomposition levels were kept [34]. After preliminary tests on our data, we decided to use marginal coefficients up to the third level obtained with 
JRRD, Volume 53, Number 3, 2016

Table 1.

Clinical characteristics of subjects with amputation.

\begin{tabular}{|c|c|c|c|c|c|c|c|c|c|c|}
\hline Subject & Handedness & $\begin{array}{c}\text { Amputated } \\
\text { Hand(s) }\end{array}$ & $\begin{array}{l}\text { Amputation } \\
\text { Cause }\end{array}$ & $\begin{array}{c}\text { Remaining } \\
\text { Forearm } \\
(\%)\end{array}$ & $\begin{array}{c}\text { Yr Since } \\
\text { Amputation }\end{array}$ & $\begin{array}{c}\text { Phantom } \\
\text { Limb } \\
\text { Sensation }\end{array}$ & $\begin{array}{c}\text { DASH } \\
\text { Score }\end{array}$ & $\begin{array}{l}\text { Performed } \\
\text { Movement }\end{array}$ & $\begin{array}{l}\text { Analyzed } \\
\text { Movement }\end{array}$ & $\begin{array}{c}\text { Used } \\
\text { Electrode }\end{array}$ \\
\hline 1 & $\overline{\mathrm{R}}$ & $\overline{\mathrm{R}}$ & A & 50 & 13 & 2 & 1.67 & 39 & 29 & 12 \\
\hline 2 & $\mathrm{R}$ & L & A & 70 & 6 & 5 & 15.18 & 50 & 40 & 12 \\
\hline 3 & $\mathrm{R}$ & $\mathrm{R}$ & A & 30 & 5 & 2 & 22.50 & 49 & 40 & 12 \\
\hline 4 & $\mathrm{R}$ & $\mathrm{R} \& \mathrm{~L}$ & A & 40 & 1 & 1 & 86.67 & 50 & 40 & 12 \\
\hline 5 & $\mathrm{~L}$ & $\mathrm{~L}$ & A & 90 & 1 & 2 & 11.67 & 50 & 40 & 12 \\
\hline 6 & $\mathrm{R}$ & $\mathrm{L}$ & A & 40 & 13 & 4 & 37.50 & 50 & 40 & 10 \\
\hline 7 & $\mathrm{R}$ & $\mathrm{R}$ & A & 0 & 7 & 0 & 31.67 & 50 & 40 & 10 \\
\hline 8 & $\mathrm{R}$ & $\mathrm{R}$ & A & 50 & 5 & 2 & 33.33 & 50 & 40 & 12 \\
\hline 9 & $\mathrm{R}$ & $\mathrm{R}$ & A & 90 & 14 & 5 & 3.33 & 50 & 40 & 12 \\
\hline 10 & $\mathrm{R}$ & $\mathrm{R}$ & A & 50 & 2 & 5 & 11.67 & 43 & 40 & 12 \\
\hline 11 & $\mathrm{R}$ & $\mathrm{R}$ & $\mathrm{C}$ & 90 & 5 & 4 & 12.50 & 50 & 40 & 12 \\
\hline Group 1 & $10 \mathrm{R}$ & $8 \mathrm{R}$ & $9 \mathrm{~A}$ & - & - & - & - & - & - & - \\
\hline Group 2 & $1 \mathrm{~L}$ & $4 \mathrm{~L}$ & $1 \mathrm{C}$ & - & - & - & - & - & - & - \\
\hline Mean & - & - & - & 54.55 & 6.55 & 2.91 & 24.33 & 48.27 & 39.00 & 11.64 \\
\hline SD & - & - & - & 28.41 & 4.78 & 1.76 & 23.87 & 3.72 & 3.32 & 0.81 \\
\hline
\end{tabular}

A = accident; C = cancer; DASH = Disabilities of the Arm, Shoulder and Hand; L = left; R = right; SD = standard deviation.

the seventh-order Daubechies wavelet [34]. The fifth feature was computed as the normalized concatenation of the others [35].

The classifiers we used are well known and have been applied in other application domains of machine learning, including sEMG analysis. They include least squares support vector machines (LSSVM) (radial basis function kernel: hyperparameters tuned for each subject by grid search with 10 -fold cross-validation) [36]; random forests (100 decision trees) [37]; support vector machines (SVM) (radial basis function kernel: hyperparameters tuned for each subject by grid search with 5-fold cross-validation) [38]; discriminant analysis (linear, naive Bayes linear, quadratic, naive Bayes quadratic, Mahalanobis) [39]; and $k$-nearest neighbors $(k-\mathrm{nn})$ $[1,15,40]$. Only low-dimensional features (RMS, WL) were used with quadratic, naive Bayes quadratic, and Mahalanobis discriminant analysis because of computational issues (i.e., singular covariance matrix).

\section{Highly Independent Movements}

Highly independent movements are defined as "a subset of movements that can be classified with accuracy above 90\%" [41]. The maximum number of highly independent movements allows us to evaluate the classification accuracies that can be reached with the same procedure on less complex tasks. Moreover, it gives an intuitive idea of the number of movements that could be controlled on a robotic hand prosthesis with enough accuracy without training (after a longer training time, we expect results to improve significantly). The analysis includes in order: a one-way multivariate analysis of variance on the training data set, the creation of a hierarchical cluster tree of the movements, the selection of subsets with increasing number of movements from the nodes of the tree, the classification of each subset of movements (the classification procedure with $k$-nn algorithm, RMS, and WL features was chosen according to computation time and memory needs; thus, we expect results to improve using several other classifiers), and the selection of the largest subset of movements with accuracy greater than 90 percent.

\section{Statistical Analysis}

The effect of clinical parameters on the results was evaluated by separately fitting the classification accuracy and independent movements computed with each classifier and each feature. Several model functions were tested, including linear functions, logarithmic functions, and the following monotonically increasing function ( $a>$ $0, b>0)$ :

$$
F(x)=a x^{b}+c
$$

The quality of the fit was evaluated by a Lilliefors test. Linear regression was applied on the transformed data, and the statistical significance of the results was evaluated with an $F$ test. 


\section{RESULTS}

\section{Movement Recognition}

The highest average classification accuracy was obtained by SVM (45.19\% $\pm 14.75 \%)$, random forests $(44.26 \% \pm 14.57 \%)$, and LSSVM $(43.17 \% \pm 14.48 \%)$, considering all the features together. The accuracy of all methods decreased by up to 4 percent when only one feature was considered; however, this reduced computation time if one-dimensional features (such as RMS and WL) were considered. The classification accuracy differed strongly between subjects: the worst result was 6.16 percent (less than 3 times the $2.5 \%$ chance level over 40 movements), while the best was 63.5 percent (more than 25 times the chance level). The average number of highly independent movements was $6.64 \pm 3.32$ with $k$-nn and RMS and $6.72 \pm 3.31$ with $k$-nn and WL. The number of highly independent movements varied strongly among subjects, from a minimum of 2 to a maximum of 11 movements. It should be noted that different subsets of movements can be selected on the basis of other parameters, such as the functional usefulness of the movements.

\section{Clinical Parameter Effects on Movement Recognition}

The classification accuracy and the number of independent movements increased significantly with phantom limb sensation intensity $(p<0.05$ in $100 \%$ of models: i.e., in 36 cases for accuracy and in 2 cases for number of independent movements; each model is computed for a unique combination of classifier and signal feature) (Figure 4(a)-(b); Table 2). The best fit was obtained in both cases with the function described in Equation 1 (respectively: $a=25.9 \pm 13.9, b=0.34 \pm$ $0.21, c=2.9 \pm 6.35, R^{2}=0.63 \pm 0.06 ; a=2.96 \pm 0.18, b=$ $\left.0.55 \pm 0.02, c=1.74 \pm 0.09, R^{2}=0.43 \pm 0.01\right)$. Considering subject 7 ( $0 \%$ of forearm remaining) as an outlier, the best fit was obtained with a logarithmic function. For classification accuracy, this result was significant $(p<$ 0.05 ) in 22.2 percent of the models, while it showed a nonsignificant trend $(p<0.1)$ in 36.1 percent of the models.

In most cases, the classification accuracy increased significantly with increased percentage of forearm remaining ( $p<0.05$ in $83.3 \%$ of analyzed models) (Figure 4(c); Table 2). Again, the best fit was obtained with the function in Equation $1(a=5.87 \pm 3.36, b=0.43 \pm$ $\left.0.14, c=9.2 \pm 3.25 ; R^{2}=0.48 \pm 0.14\right)$. Considering subject 7 ( $0 \%$ of forearm remaining) as an outlier, the best fit was obtained with a logarithmic function; this result was significant $(p<0.05)$ in 5.6 percent of models, while it showed a nonsignificant trend $(p<0.1)$ in 25 percent of the models. In 67 percent of the cases, the number of highly independent movements showed an increasing trend versus remaining forearm percentage $(p<0.1)$. Considering subject 7 ( $0 \%$ of forearm remaining) as an outlier, the classification accuracy and the number of independent movements increased significantly with time since amputation $(p<0.05$ in $100 \%$ of models for accuracy; $p<0.01$ in two models for highly independent movements) (Figure 4(e)-(f); Table 2). The results were obtained with linear fits (respectively: slope $=1.45 \pm 0.25$, intercept $=27.75 \pm 8.01, R^{2}=0.60 \pm 0.09$; slope $=0.577 \pm$ 0.008 , intercept $=3.40 \pm 0.02, R^{2}=0.88 \pm 0.03$ ). Similar trends were also obtained when subject 7 was included in the analyses; however, often with evidence for rejecting the null hypothesis of normality of the residuals.

In one case, the number of highly independent movements significantly decreased with increasing DASH score $(p<0.05)$ according to a logarithmic function. Regression analysis was also performed on the other clinical parameters, but no other significant dependencies were found among the parameters in Figure 5 and Table 2. In addition, no significant relationships were obtained considering unbalanced classification results.

\section{DISCUSSION}

Currently, myoelectric prostheses enable subjects with transradial amputation to perform few movements using pattern-recognition techniques. In recent years, the scientific literature (and one commercial product) have proposed significant improvements over the conventional myoelectric control strategy. These results can be improved further by considering a description of the problem that includes the effect of clinical parameters related to the amputation.

In this article, we analyzed the largest database of sEMG recordings of hand movements performed by subjects with transradial amputation, focusing on the relationship between clinical parameters and movement classification accuracy. The database is publicly available, and we hope that it will lead to further improvements in techniques by permitting worldwide research groups to test new machine learning algorithms on the same data in the same setting. The analysis of the database reveals interesting results that can potentially improve the quality of life and the prognosis of subjects with transradial amputation. 
JRRD, Volume 53, Number 3, 2016
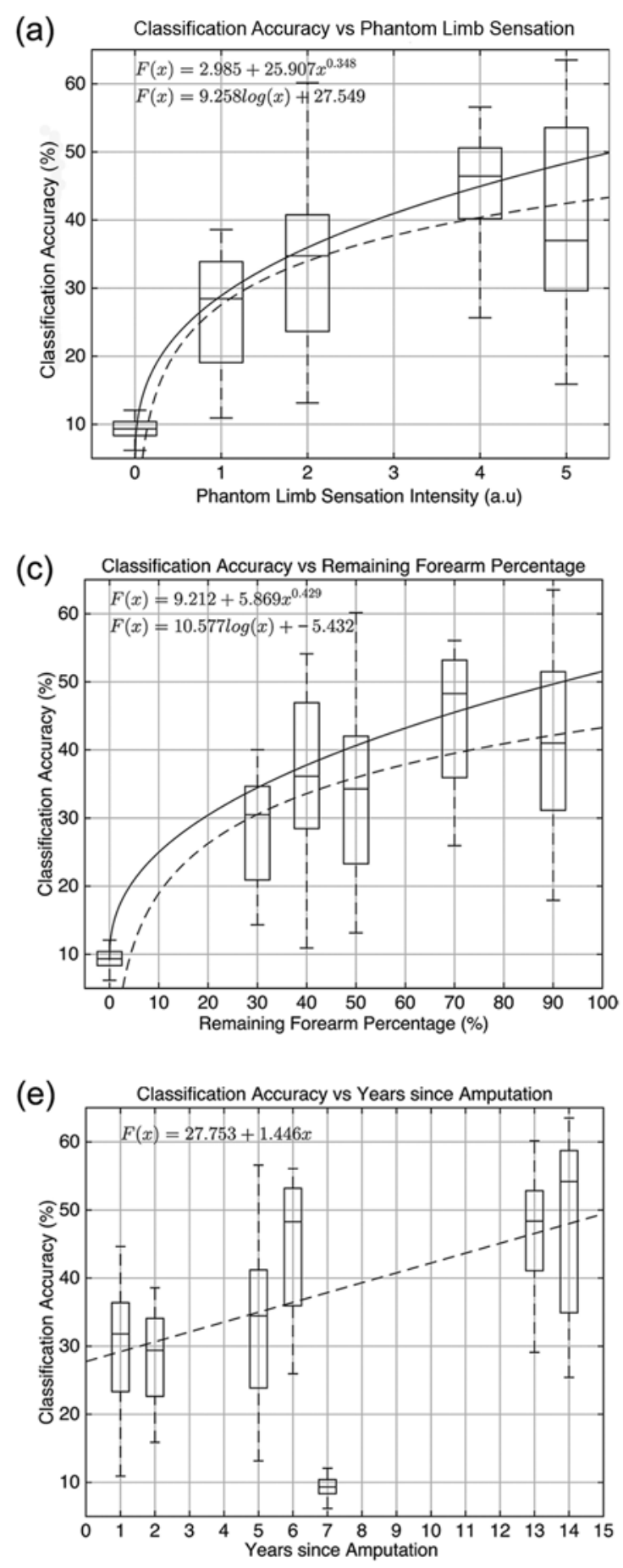
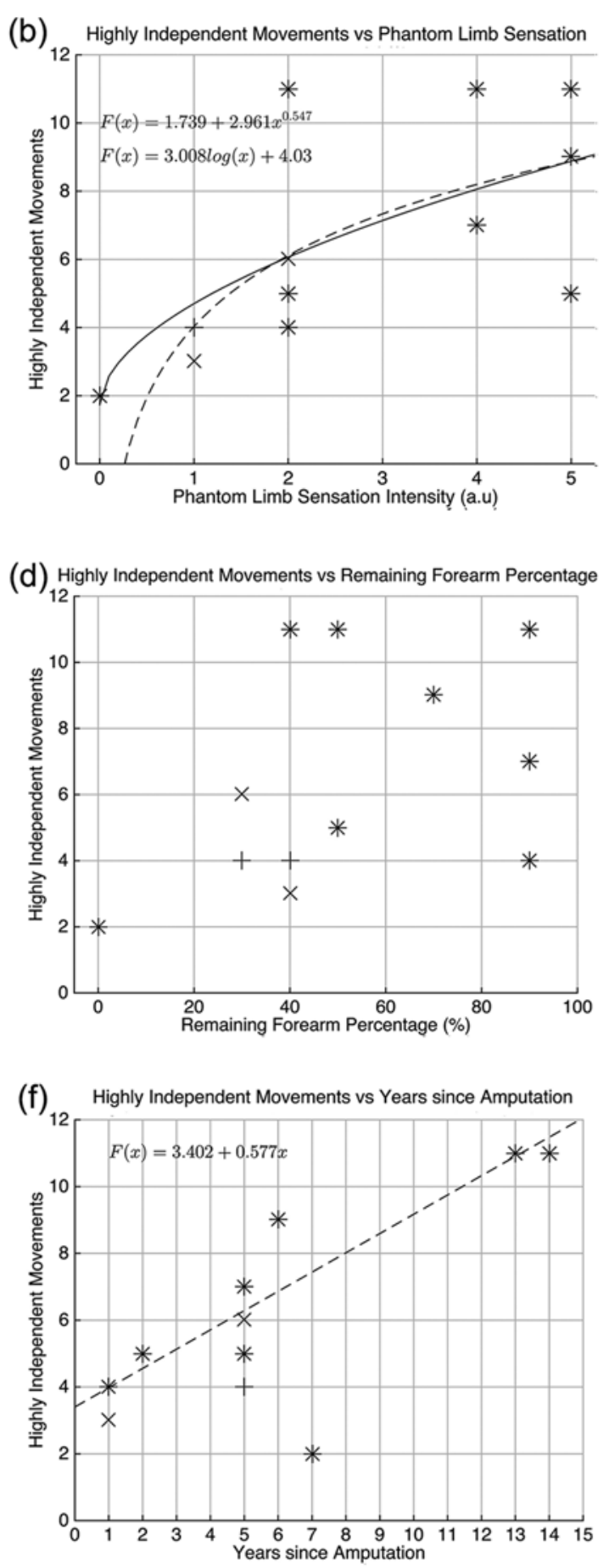

Figure 4.

Dependency of ((a), (c), (e)) average classification accuracy and ((b), (d), (f)) highly independent movements on clinical parameters. The central mark in the boxes is the median; the edges of the box are the 25th and 75th percentiles; the whiskers extend to approximately 2.7 times the standard deviation. The " $X$ " and cross markers represent the number of highly independent movements obtained using $k$-nearest neighbor with root mean square (cross markers) and waveform length ("X" markers) for each subject. The continuous fit line and the (first, when there are more than one) formula represent the fit average results with all the data. The dotted fit line and the (second, when there are more than one) formula represent the average fit without outliers. 
Table 2.

Statistical significance analysis for the relationship between classification accuracy and clinical parameters with each classifier-feature combination. In bold: $p<0.05$; in bold and italic: $0.05<p<0.1$.

\begin{tabular}{|c|c|c|c|c|c|c|c|c|c|c|c|c|c|c|c|c|}
\hline \multicolumn{2}{|c|}{ Classification Accuracy } & \multicolumn{3}{|c|}{$\begin{array}{c}\text { Phantom Limb } \\
\text { Sensation* }\end{array}$} & \multicolumn{3}{|c|}{$\begin{array}{c}\text { Phantom Limb } \\
\text { Sensation }^{\dagger}\end{array}$} & \multicolumn{3}{|c|}{$\begin{array}{c}\text { Remaining Forearm } \\
\text { Percentage* }^{*}\end{array}$} & \multicolumn{3}{|c|}{$\begin{array}{c}\text { Remaining Forearm } \\
\text { Percentage }^{\dagger}\end{array}$} & \multicolumn{3}{|c|}{$\begin{array}{c}\text { Yr Since } \\
\text { Amputation }^{\dagger}\end{array}$} \\
\hline Classifier & Feature & LT & $R^{2}$ & LR & LT & $R^{2}$ & LR & $\mathbf{L T}$ & Fit $R^{2}$ & LR & LT & $R^{2}$ & LR & LT & Fit $R^{2}$ & LR \\
\hline \multirow[t]{5}{*}{ SVM } & All & 0.267 & 0.700 & 0.001 & 0.500 & 0.289 & 0.109 & 0.500 & 0.686 & 0.001 & 0.500 & 0.338 & 0.078 & 0.500 & 0.557 & 0.013 \\
\hline & HIST & 0.107 & 0.636 & 0.003 & 0.097 & 0.266 & 0.127 & 0.500 & 0.663 & 0.001 & 0.500 & 0.360 & 0.067 & 0.500 & 0.489 & 0.024 \\
\hline & RMS & 0.500 & 0.663 & 0.002 & 0.500 & 0.220 & 0.172 & 0.500 & 0.433 & 0.004 & 0.500 & 0.108 & 0.354 & 0.114 & 0.773 & 0.001 \\
\hline & WL & 0.473 & 0.706 & 0.001 & 0.500 & 0.201 & 0.194 & 0.500 & 0.560 & 0.001 & 0.500 & 0.195 & 0.202 & 0.500 & 0.680 & 0.003 \\
\hline & mDWT & 0.500 & 0.685 & 0.002 & 0.500 & 0.269 & 0.125 & 0.500 & 0.532 & 0.003 & 0.500 & 0.206 & 0.187 & 0.339 & 0.701 & 0.003 \\
\hline \multirow[t]{5}{*}{ LSSVM } & All & 0.455 & 0.680 & 0.002 & 0.500 & 0.284 & 0.113 & 0.500 & 0.642 & 0.001 & 0.500 & 0.247 & 0.144 & 0.500 & 0.629 & 0.006 \\
\hline & HIST & 0.204 & 0.625 & 0.004 & 0.435 & 0.272 & 0.122 & 0.500 & 0.599 & 0.002 & 0.225 & 0.356 & 0.069 & 0.500 & 0.580 & 0.011 \\
\hline & RMS & 0.500 & 0.647 & 0.003 & 0.500 & 0.232 & 0.159 & 0.500 & 0.386 & 0.006 & 0.500 & 0.206 & 0.187 & 0.500 & 0.745 & 0.001 \\
\hline & WL & 0.170 & 0.660 & 0.002 & 0.214 & 0.170 & 0.236 & 0.500 & 0.523 & 0.002 & 0.476 & 0.208 & 0.185 & 0.500 & 0.655 & 0.005 \\
\hline & mDWT & 0.500 & 0.632 & 0.003 & 0.500 & 0.233 & 0.157 & 0.500 & 0.527 & 0.004 & 0.500 & 0.272 & 0.122 & 0.500 & 0.686 & 0.003 \\
\hline \multirow[t]{5}{*}{ Random Forests } & All & 0.463 & 0.684 & 0.002 & 0.351 & 0.239 & 0.151 & 0.500 & 0.632 & 0.002 & 0.281 & 0.388 & 0.054 & 0.500 & 0.677 & 0.003 \\
\hline & HIST & 0.312 & 0.664 & 0.002 & 0.500 & 0.246 & 0.145 & 0.500 & 0.649 & 0.001 & 0.442 & 0.456 & 0.032 & 0.500 & 0.568 & 0.012 \\
\hline & RMS & 0.446 & 0.698 & 0.001 & 0.500 & 0.313 & 0.093 & 0.500 & 0.596 & 0.003 & 0.500 & 0.113 & 0.343 & 0.500 & 0.720 & 0.002 \\
\hline & WL & 0.500 & 0.676 & 0.002 & 0.500 & 0.220 & 0.171 & 0.500 & 0.557 & 0.002 & 0.500 & 0.203 & 0.191 & 0.500 & 0.672 & 0.004 \\
\hline & mDWT & 0.500 & 0.669 & 0.002 & 0.341 & 0.256 & 0.135 & 0.500 & 0.614 & 0.002 & 0.353 & 0.173 & 0.232 & 0.380 & 0.641 & 0.005 \\
\hline \multirow[t]{5}{*}{$k$-nn } & All & 0.076 & 0.622 & 0.004 & 0.149 & 0.285 & 0.112 & 0.500 & 0.580 & 0.003 & 0.072 & 0.090 & 0.399 & 0.500 & 0.583 & 0.010 \\
\hline & HIST & 0.039 & 0.611 & 0.005 & 0.129 & 0.276 & 0.119 & 0.500 & 0.615 & 0.002 & 0.030 & 0.120 & 0.326 & 0.500 & 0.520 & 0.019 \\
\hline & RMS & 0.500 & 0.621 & 0.004 & 0.500 & 0.240 & 0.151 & 0.500 & 0.416 & 0.008 & 0.014 & 0.030 & 0.633 & 0.075 & 0.762 & 0.001 \\
\hline & WL & 0.500 & 0.646 & 0.003 & 0.500 & 0.204 & 0.190 & 0.500 & 0.514 & 0.004 & 0.090 & 0.037 & 0.593 & 0.500 & 0.708 & 0.002 \\
\hline & mDWT & 0.500 & 0.636 & 0.003 & 0.500 & 0.272 & 0.122 & 0.500 & 0.571 & 0.003 & 0.047 & 0.119 & 0.330 & 0.500 & 0.582 & 0.010 \\
\hline \multirow[t]{4}{*}{ Linear } & HIST & 0.056 & 0.627 & 0.004 & 0.077 & 0.397 & 0.051 & 0.500 & 0.527 & 0.006 & 0.138 & 0.164 & 0.245 & 0.500 & 0.517 & 0.019 \\
\hline & RMS & 0.144 & 0.587 & 0.006 & 0.239 & 0.364 & 0.065 & 0.494 & 0.135 & 0.049 & 0.123 & 0.202 & 0.192 & 0.500 & 0.571 & 0.011 \\
\hline & WL & 0.391 & 0.613 & 0.004 & 0.489 & 0.370 & 0.062 & 0.321 & 0.163 & 0.035 & 0.350 & 0.192 & 0.205 & 0.500 & 0.548 & 0.014 \\
\hline & mDWT & 0.311 & 0.599 & 0.005 & 0.500 & 0.274 & 0.120 & 0.500 & 0.230 & 0.016 & 0.500 & 0.363 & 0.065 & 0.344 & 0.653 & 0.005 \\
\hline \multirow[t]{4}{*}{ Naive Bayes Linear } & HIST & 0.237 & 0.478 & 0.018 & 0.255 & 0.399 & 0.049 & 0.182 & 0.459 & 0.118 & 0.500 & 0.422 & 0.042 & 0.500 & 0.473 & 0.028 \\
\hline & RMS & 0.500 & 0.482 & 0.018 & 0.500 & 0.399 & 0.049 & 0.213 & 0.469 & 0.101 & 0.389 & 0.121 & 0.325 & 0.500 & 0.426 & 0.041 \\
\hline & WL & 0.002 & 0.502 & 0.015 & 0.006 & 0.442 & 0.036 & 0.022 & 0.304 & 0.201 & 0.500 & 0.161 & 0.251 & 0.500 & 0.477 & 0.027 \\
\hline & mDWT & 0.015 & 0.499 & 0.015 & 0.024 & 0.407 & 0.047 & 0.105 & 0.403 & 0.155 & 0.500 & 0.323 & 0.087 & 0.500 & 0.513 & 0.020 \\
\hline \multirow[t]{2}{*}{ Quadr. } & RMS & 0.295 & 0.615 & 0.004 & 0.363 & 0.279 & 0.116 & 0.500 & 0.529 & 0.005 & 0.405 & 0.002 & 0.893 & 0.179 & 0.697 & 0.003 \\
\hline & WL & 0.370 & 0.641 & 0.003 & 0.348 & 0.287 & 0.110 & 0.500 & 0.584 & 0.003 & 0.500 & 0.010 & 0.786 & 0.171 & 0.636 & 0.006 \\
\hline \multirow[t]{3}{*}{ Naive Bayes Quadr. } & RMS & 0.373 & 0.604 & 0.005 & 0.500 & 0.530 & 0.017 & 0.232 & 0.278 & 0.063 & 0.500 & 0.057 & 0.506 & 0.500 & 0.511 & 0.020 \\
\hline & WL & 0.291 & 0.667 & 0.002 & 0.500 & 0.577 & 0.011 & 0.096 & 0.338 & 0.035 & 0.500 & 0.198 & 0.198 & 0.500 & 0.471 & 0.028 \\
\hline & mDWT & 0.500 & 0.681 & 0.002 & 0.500 & 0.565 & 0.012 & 0.383 & 0.341 & 0.030 & 0.500 & 0.261 & 0.131 & 0.279 & 0.499 & 0.022 \\
\hline \multirow[t]{2}{*}{ Mahal. } & RMS & 0.500 & 0.596 & 0.005 & 0.500 & 0.312 & 0.093 & 0.500 & 0.447 & 0.011 & 0.500 & 0.229 & 0.162 & 0.500 & 0.742 & 0.001 \\
\hline & WL & 0.500 & 0.637 & 0.003 & 0.500 & 0.289 & 0.109 & 0.500 & 0.580 & 0.004 & 0.399 & 0.250 & 0.141 & 0.500 & 0.659 & 0.004 \\
\hline \multirow{2}{*}{\multicolumn{2}{|c|}{ Independent Movements }} & \multicolumn{3}{|c|}{$\begin{array}{c}\text { Phantom Limb } \\
\text { Sensation* }\end{array}$} & \multicolumn{3}{|c|}{$\begin{array}{c}\text { Phantom Limb } \\
\text { Sensation }^{\dagger}\end{array}$} & \multicolumn{3}{|c|}{$\begin{array}{c}\text { Yr Since } \\
\text { Amputation }^{\dagger} \\
\end{array}$} & \multicolumn{3}{|c|}{ DASH Score ${ }^{*}$} & & & \\
\hline & & LT & $R^{2}$ & LR & LT & $R^{2}$ & LR & LT & $R^{2}$ & LR & LT & $R^{2}$ & LR & & & \\
\hline$k$-nn & MS & 0.240 & 0.423 & 0.030 & 0.500 & 0.277 & 0.118 & 0.218 & 0.858 & $<10^{-3}$ & 0.500 & 0.237 & 0.061 & - & - & - \\
\hline & VL & 0.332 & 0.437 & 0.027 & 0.350 & 0.306 & 0.097 & 0.351 & 0.904 & $<10^{-3}$ & 0.349 & 0.37 & 0.047 & - & - & - \\
\hline
\end{tabular}

All data.

${ }^{\dagger}$ Without subject 7.

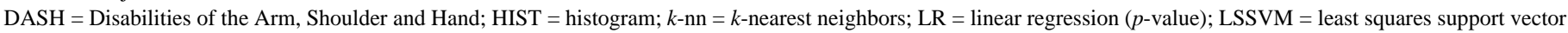

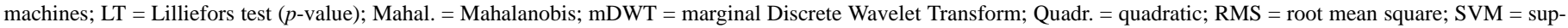
port vector machines; $\mathrm{WL}=$ waveform length.

\section{Movement Recognition}

Depending on the characteristics of the subjects in our sample, we found that it was possible to reach up to 63.5 percent balanced accuracy in the recognition of 40 movements. This result is much lower than what can be found in other studies, but in our case the chance level is a mere 2.5 percent because of the high number of classes (movements) considered. In particular, the ratio between the accuracy and the chance level in our result (25.4) is higher than in previous studies described in the literature for similar tasks, for example, 8.5 (10 movements, accuracy $84.4 \%$ [6]) or 10.56 (12 movements, accuracy $87.8 \%$ [5]). An extended explanation of this together with a direct comparison of the classification results with previous work in a similar setting is described in Atzori et al. [19]. 
JRRD, Volume 53, Number 3, 2016
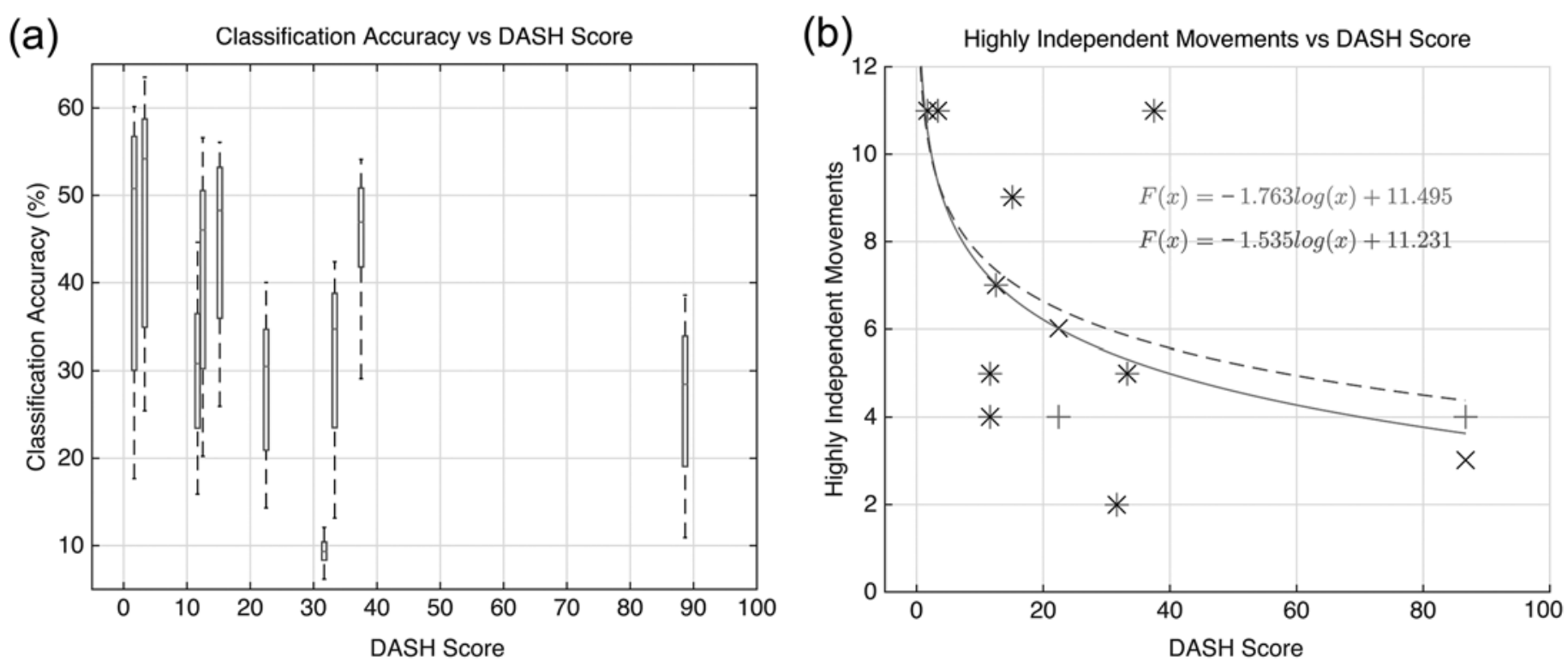

Figure 5.

Dependency of (a) average classification accuracy and (b) highly independent movements on the Disabilities of the Arm, Shoulder and Hand (DASH) score. The central mark in the boxes is the median; the edges of the box are the 25th and 75th percentiles; the whiskers extend to approximately 2.7 times the standard deviation. The "X" and cross markers represent the number of highly independent movements obtained using $k$-nearest neighbor with root mean square (cross markers) and waveform length ("X" markers) for each subject. The continuous fit line and the first formula represent the fit average results with all the data. The dotted fit line and the second formula represent the average fit without outliers.

Our results show that it is possible to find up to 11 highly independent movements in three subjects and, that for a few movements, very high classification accuracy is possible through training the control system rather than the subjects. In particular, this strategy can be applied in a practical setting to make subjects with transradial amputation able to naturally control an average of 6 movements, a number sufficient for most everyday tasks, for robotic, dexterous hand prostheses with no prior experience. If needed, a longer training of both the patient and the system could further improve the results; in addition, most of the subjects reported at the end of the acquisition that they had increased their ability to reproduce the movements.

\section{Effects of Clinical Parameters on Movement Recognition}

The classification accuracy and the number of independent movements seem to depend mainly on three parameters: phantom limb sensation intensity, remaining forearm percentage, and time since amputation. This result proved to be significant in both cases for phantom limb sensation intensity (Figure 4(a)-(b)), denoting sig- nificant interaction between the clinical condition of the subjects and the ability to control remnant muscles. This information can help clarify the (still unclear) nature of this syndrome, which is strongly related to phantom limb pain [42] and affects more than 70 percent of persons with amputation [43].

The significant dependence of classification accuracy on the remaining forearm percentage (Figure 4(c)) seems reasonable considering that the percentage of remaining muscles depends strictly on the percentage of remaining forearm. This result can seem obvious, but it has not been described in literature previously and it contributes to the importance of considering muscular flaps as possible input sources for multiple sensors.

Both the classification accuracy and the number of independent movements increased with time since amputation (Figure 4(e)-(f)). This result shows that the subjects are still capable of controlling the muscles despite the cortical reorganization that takes place after amputation, in accordance with what is described for the somatosensory path $[14,44]$. Moreover, this result demonstrates that even subjects who underwent amputation decades ago 
should be able to gain advantages related to robotic dexterous prostheses.

Despite the number of subjects in this study being relatively high compared with other studies, in an absolute sense it is not. The decision of including subject 7 ( $0 \%$ of forearm remaining) in the analysis was driven by the idea of not removing an important data point as well as to give a better perspective on all the levels of amputation. However, we should note that when considering the subject as an outlier, the percentage of significant results decreased. This fact suggests that the presented results should be discussed in future studies performed on a higher number of subjects.

\section{Clinical Implications}

Amputation of the upper limb is one of the most severely impairing injuries: clinical implications of these outcomes are substantial and have the potential to improve the quality of life and prognosis for these patients by improving the rehabilitation capabilities offered by modern prostheses.

The relationship between movement classification accuracy and phantom limb sensation suggests that amputated subjects with high phantom limb sensation can control the remnant muscles of the amputated hand better than people with low phantom limb sensation. The reasons behind this result should be explored in future studies. To our knowledge, this result has never been observed before; thus, it can contribute to improving knowledge of phantom limb sensation, which still has an unclear etiology.

The significant relationships between classification accuracy and phantom limb sensation, remaining forearm percentage, and time since amputation can contribute to identifying surgical guidelines that optimize the expected control capabilities of modern adaptive prostheses. Currently tailored to adapt to standard prosthetic devices, "conservative" amputations and reconstructive surgical strategies can be improved to better integrate with dexterous functional prosthetic hands using current machine learning approaches and the most efficient rehabilitation programs in the near future. Integration with an advanced prosthesis can be the first step in the treatment of a patient with an amputated arm, rather than the final step. The three identified relationships suggest that muscular flaps are likely to evolve from being only soft tissue coverage and material for fitting the residual limb to acting as a highly specific and sophisticated input source of data for sEMG learning hand prostheses ("functional amputation”). Such procedures can be as effective as targeted muscle reinnervation, while being at the same time less invasive, in particular considering the positive effect of time on the movement recognition capabilities. Consistently, being less dependent on individual training, rehabilitative strategies are expected to achieve higher performance and a more intuitive control of reasonably complex tasks. This approach can also target economic limits or lack of available rehabilitative resources, affecting a larger percentage of patients, including those unable to obtain adequate rehabilitative assistance for physical reasons (presence of exercise-related fatigue and pain, lack of patient compliance, presence of phantom limb syndrome) who could benefit from these advances.

\section{CONCLUSIONS}

The amputation of the upper limb is one of the most severely impairing injuries. As described in the scientific literature, sEMG is a promising method to control noninvasive, dexterous, robotic prosthetic hands. The application of machine learning to the analysis of sEMG is promising and recently has been applied in practice, but many questions still remain because of the heterogeneity of the studies and lack of data. This fact contributes to the lack of studies analyzing the effects of clinical parameters, which can improve rehabilitation of the patients.

In this article, we analyzed the largest (publicly available) database of sEMG recordings of hand movements performed by subjects with amputation, focusing on the relationship between clinical parameters and movement classification accuracy. We found that there are significant relationships between movement recognition performance and clinical parameters, such as phantom limb sensation intensity, amputation level, and time since amputation. The number of subjects is high compared with other studies; however, it is not high in absolute terms. This fact suggests that the presented results should be discussed in future studies performed on a higher number of subjects.

In any case, the proposed analyses have the potential to improve quality of life and prognosis for subjects with amputation in two main ways. First, the prostheses can be improved and adapted to the clinical characteristics of the subjects. Second, the relationship between movement classification accuracy and the three clinical parameters 
can contribute to identifying surgical guidelines for "functional amputation" procedures aimed at optimizing prosthesis integration and improving rehabilitative capabilities.

\section{ACKNOWLEDGMENTS}

\author{
Author Contributions: \\ Created initial project: C. Castellini. \\ Wrote initial project: $\mathrm{H}$. Müller. \\ Wrote and coordinated project: B. Caputo. \\ Recruited subjects: S. Elsig, G. Giatsidis, F. Bassetto. \\ Created data acquisition software: A. Gijsberts, C. Castellini. \\ Created data acquisition protocol: M. Atzori, A. M. Hager, S. Elsig. \\ Data acquisition: M. Atzori, A. M. Hager, S. Elsig, G. Giatsidis, \\ F. Bassetto. \\ Data analysis: M. Atzori. \\ Drafted manuscript: M. Atzori. \\ Critical revision of manuscript for important intellectual content: \\ A. Gijsberts, C. Castellini, B. Caputo, A. M. Hager, S. Elsig, \\ G. Giatsidis, F. Bassetto, H. Müller. \\ Supervised the project: H. Müller.
}

Financial Disclosures: The authors have declared that no competing interests exist.

Funding/Support: This material was based on work supported by the Swiss National Science Foundation (Sinergia) (grant 132700).

Additional Contributions: We would like to thank the people who volunteered for this project.

Institutional Review: The experiment was conducted according to the principles of the Declaration of Helsinki. It was approved by the Ethics Commission of the state of Valais (Switzerland), and all participants signed an informed consent form.

Participant Follow-Up: The authors plan to notify the study subjects of the publication of this article.

\section{REFERENCES}

1. Atkins DJ, Heard DC, Donovan WH. Epidemiologic overview of individuals with upper-limb loss and their reported research priorities. J Prosthet Orthot. 1996;8(1):2-11. http://dx.doi.org/10.1097/00008526-199600810-00003

2. Castellini C, Gruppioni E, Davalli A, Sandini G. Fine detection of grasp force and posture by amputees via surface electromyography. J Physiol Paris. 2009;103(3-5):25562. [PMID:19665563]

http://dx.doi.org/10.1016/j.jphysparis.2009.08.008

3. Farrell TR, Weir RF. A comparison of the effects of electrode implantation and targeting on pattern classification accuracy for prosthesis control. IEEE Trans Biomed Eng. 2008;55(9):2198-211. [PMID:18713689] http://dx.doi.org/10.1109/TBME.2008.923917

4. Crawford B, Miller K, Shenoy P, Rao R. Real-time classification of electromyographic signals for robotic control.
Proceedings of the 20th National Conference on Artificial Intelligence; 2005 Jul; Pittsburgh, PA. Palo Alto (CA): AAAI Press. p. 523-28.

5. Tenore FV, Ramos A, Fahmy A, Acharya S, EtienneCummings R, Thakor NV. Decoding of individuated finger movements using surface electromyography. IEEE Trans Biomed Eng. 2009;56(5):1427-34. [PMID:19473933] http://dx.doi.org/10.1109/TBME.2008.2005485

6. Li G, Schultz AE, Kuiken TA. Quantifying pattern recognition-based myoelectric control of multifunctional transradial prostheses. IEEE Trans Neural Syst Rehabil Eng. 2010; 18(2):185-92. [PMID:20071269]

http://dx.doi.org/10.1109/TNSRE.2009.2039619

7. Cipriani C, Antfolk C, Controzzi M, Lundborg G, Rosen B, Carrozza MC, Sebelius F. Online myoelectric control of a dexterous hand prosthesis by transradial amputees. IEEE Trans Neural Syst Rehabil Eng. 2011;19(3):260-70.

[PMID:21292599]

http://dx.doi.org/10.1109/TNSRE.2011.2108667

8. Peerdeman B, Boere D, Witteveen H, in 't Veld RH, Hermens H, Stramigioli S, Rietman H, Veltink P, Misra S. Myoelectric forearm prostheses: State of the art from a user-centered perspective. J Rehabil Res Dev. 2011;48(6): 719-37. [PMID:21938658] http://dx.doi.org/10.1682/JRRD.2010.08.0161

9. Kuiken TA, Li G, Lock BA, Lipschutz RD, Miller LA, Stubblefield KA, Englehart KB. Targeted muscle reinnervation for real-time myoelectric control of multifunction artificial arms. JAMA. 2009;301(6):619-28.

[PMID:19211469]

http://dx.doi.org/10.1001/jama.2009.116

10. Raspopovic S, Capogrosso M, Petrini FM, Bonizzato M, Rigosa J, Di Pino G, Carpaneto J, Controzzi M, Boretius T, Fernandez E, Granata G, Oddo CM, Citi L, Ciancio AL, Cipriani C, Carrozza MC, Jensen W, Guglielmelli E, Stieglitz T, Rossini PM, Micera S. Restoring natural sensory feedback in real-time bidirectional hand prostheses. Sci Transl Med. 2014;6(222):222ra19. [PMID:24500407] http://dx.doi.org/10.1126/scitranslmed.3006820

11. Borton D, Micera S, Millán JR, Courtine G. Personalized neuroprosthetics. Sci Transl Med. 2013;5(210):210rv2.

[PMID:24197737] http://dx.doi.org/10.1126/scitranslmed.3005968

12. Naik GR, Kumar DK, Jayadeva. Twin SVM for gesture classification using the surface electromyogram. IEEE Trans Inf Technol Biomed. 2010;14(2):301-08. [PMID:20007054] http://dx.doi.org/10.1109/TITB.2009.2037752

13. Sebelius FC, Rosén BN, Lundborg GN. Refined myoelectric control in below-elbow amputees using artificial neural networks and a data glove. J Hand Surg Am. 2005;30(4): 
780-89. [PMID:16039372]

http://dx.doi.org/10.1016/j.jhsa.2005.01.002

14. Schwenkreis P, Witscher K, Janssen F, Pleger B, Dertwinkel R, Zenz M, Malin JP, Tegenthoff M. Assessment of reorganization in the sensorimotor cortex after upper limb amputation. Clin Neurophysiol. 2001;112(4):627-35.

[PMID:11275535]

http://dx.doi.org/10.1016/S1388-2457(01)00486-2

15. He H, Garcia E. Learning from imbalanced data. IEEE Trans Knowl Data Eng. 2009;21(9):1263-84.

http://dx.doi.org/10.1109/TKDE.2008.239

16. Atzori M, Gijsberts A, Kuzborskij I, Elsig S, Hager AG, Deriaz O, Castellini C, Muller H, Caputo B. Characterization of a benchmark database for myoelectric movement classification. IEEE Trans Neural Syst Rehabil Eng. 2015; 23(1):73-83. [PMID:25486646]

http://dx.doi.org/10.1109/TNSRE.2014.2328495

17. Hudak PL, Amadio PC, Bombardier C; The Upper Extremity Collaborative Group (UECG). Development of an upper extremity outcome measure: The DASH (disabilities of the arm, shoulder and hand) [corrected]. Am J Ind Med. 1996;29(6):602-8. [PMID:8773720] http://dx.doi.org/10.1002/(SICI)1097-0274(199606)29:6<602 ::AID-AJIM4>3.0.CO;2-L

18. Von Korff M, Ormel J, Keefe FJ, Dworkin SF. Grading the severity of chronic pain. Pain. 1992;50(2):133-49.

[PMID:1408309] http://dx.doi.org/10.1016/0304-3959(92)90154-4

19. Atzori M, Gijsberts A, Castellini C, Caputo B, Hager AG, Elsig S, Giatsidis G, Bassetto F, Müller H. Electromyography data for non-invasive naturally-controlled robotic hand prostheses. Sci Data. 2014;1:140053. [PMID:25977804] http://dx.doi.org/10.1038/sdata.2014.53

20. Gijsberts A, Atzori M, Castellini C, Muller H, Caputo B. Movement error rate for evaluation of machine learning methods for sEMG-based hand movement classification. IEEE Trans Neural Syst Rehabil Eng. 2014;22(4):735-44. [PMID:24760932] http://dx.doi.org/10.1109/TNSRE.2014.2303394

21. Feix T, Romero J, Schmiedmayer HB, Dollar AM, Kragic D. The GRASP taxonomy of human grasp types. IEEE Trans Hum Mach Sys. 2016;46(1):66-77. http://dx.doi.org/10.1109/THMS.2015.2470657

22. Kamakura N, Matsuo M, Ishii H, Mitsuboshi F, Miura Y. Patterns of static prehension in normal hands. Am J Occup Ther. 1980;34(7):437-45. [PMID:6446851] http://dx.doi.org/10.5014/ajot.34.7.437

23. Cutkosky MR. On grasp choice, grasp models, and the design of hands for manufacturing tasks. IEEE Trans Rob Autom. 1989;5(3):269-79. http://dx.doi.org/10.1109/70.34763
24. Edwards SJ, Buckland DJ, McCoy-Powlen JD. Developmental and functional hand grasps. Thorofare (NJ): Slack Incorporated; 2002.

25. Kato R, Yokoi H, Arai T. Competitive learning method for robust EMG-to-motion classifier. 9th International Conference on Intelligent Autonomous Systems, IAS 2006; 2006 Mar 7-9; Tokyo, Japan. Amsterdam (The Netherlands): IOS Press; 2006. p. 946-53.

26. Fukuda O, Tsuji T, Kaneko M, Otsuka A, Tsuji OF. A human-assisting manipulator teleoperated by EMG signals and arm motions. IEEE Trans Rob Autom. 2003;19(2): 210-22. http://dx.doi.org/10.1109/TRA.2003.808873

27. De Luca CJ. The use of surface electromyography in biomechanics. J Appl Biomech. 1997;13(2):135-63.

28. Castellini C, Fiorilla AE, Sandini G. Multi-subject/daily-life activity EMG-based control of mechanical hands. J Neuroeng Rehabil. 2009;6(41):41. [PMID:19919710]

http://dx.doi.org/10.1186/1743-0003-6-41

29. Englehart K, Hudgins B. A robust, real-time control scheme for multifunction myoelectric control. IEEE Trans Biomed Eng. 2003;50(7):848-54. [PMID:12848352] http://dx.doi.org/10.1109/TBME.2003.813539

30. Englehart K, Hudgins B, Parker PA, Stevenson M. Classification of the myoelectric signal using time-frequency based representations. Med Eng Phys. 1999;21(6-7):431-38. PMID:10624739] http://dx.doi.org/10.1016/S1350-4533(99)00066-1

31. Kuzborskij I, Gijsberts A, Caputo B. On the challenge of classifying 52 hand movements from surface electromyography. 2012 Annual International Conference of the IEEE Engineering in Medicine and Biology Society (EMBC); 2012 Aug 28-Sep 1; San Diego, CA. Piscataway (NJ): IEEE; 2012. p. 4931-7.

32. Tkach D, Huang H, Kuiken TA. Study of stability of timedomain features for electromyographic pattern recognition.

J Neuroeng Rehabil. 2010;7:21. [PMID:20492713] http://dx.doi.org/10.1186/1743-0003-7-21

33. Zardoshti-Kermani M, Wheeler BC, Badie K, Hashemi RM. EMG feature evaluation for movement control of upper extremity prostheses. IEEE Trans Rehabil Eng. 1995; 3(4):324-33. http://dx.doi.org/10.1109/86.481972

34. Lucas M, Gaufriau A, Pascual S, Doncarli C, Farina D. Multi-channel surface EMG classification using support vector machines and signal-based wavelet optimization. Biomed Signal Process Control. 2008;3(2):169-74. http://dx.doi.org/10.1016/j.bspc.2007.09.002

35. Fougner A, Scheme E, Chan AD, Englehart K, Stavdahl Ø. A multi-modal approach for hand motion classification using surface EMG and accelerometers. 2011 Annual International Conference of the IEEE Engineering in Medicine and 
Biology Society (EMBC); 2011 Aug 30-Sep 3; Boston, MS. Piscataway (NJ): IEEE; 2011. p. 4247-50.

36. Suykens JA, Van Gestel T, De Brabanter J, De Moor B, Vandewalle J. Least squares support vector machines. Singapore: World Scientific; 2002. 308 p.

37. Breiman L. Random forests. Mach Learn. 2001;45:5-32. http://dx.doi.org/10.1023/A:1010933404324

38. Cristianini N, Shawe-Taylor J. An introduction to support vector machines and other kernel-based learning methods. Cambridge (UK): Cambridge University Press; 2000.

39. Krzanowski WJ, Hand DJ. Principles of multivariate analysis: A user's perspective. Oxford (UK): Oxford University Press; 1988.

40. Duda RO, Hart P. Pattern classification. Hoboken (NJ): Wiley-Interscience; 2001.

41. Atzori M, Gijsberts A, Caputo B, Müller H. Natural control capabilities of robotic hands by hand amputated subjects. 2014 36th Annual International Conference of the IEEE Engineering in Medicine and Biology Society (EMBC); 2014 Aug 26-30; Chicago, IL. Piscataway (NJ): IEEE; 2014. p. 4362-65.

42. Kooijman CM, Dijkstra PU, Geertzen JH, Elzinga A, van der Schans CP. Phantom pain and phantom sensations in upper limb amputees: An epidemiological study. Pain. 2000; 87(1):33-41. [PMID:10863043] http://dx.doi.org/10.1016/S0304-3959(00)00264-5

43. Sherman RA, Sherman CJ, Parker L. Chronic phantom and stump pain among American veterans: Results of a survey. Pain. 1984;18(1):83-95. [PMID:6709380] http://dx.doi.org/10.1016/0304-3959(84)90128-3
44. Schady W, Braune S, Watson S, Torebjörk HE, Schmidt R. Responsiveness of the somatosensory system after nerve injury and amputation in the human hand. Ann Neurol. 1994;36(1):68-75. [PMID:8024265] http://dx.doi.org/10.1002/ana.410360114

Submitted for publication September 18, 2014. Accepted in revised form July 15, 2015.

This article and any supplementary material should be cited as follows:

Atzori M, Gijsberts A, Castellini C, Caputo B, Hager AM, Elsig S, Giatsidis G, Bassetto F, Müller H. Effect of clinical parameters on the control of myoelectric robotic prosthetic hands. J Rehabil Res Dev. 2016;53(3):345-58. http://dx.doi.org/10.1682/JRRD.2014.09.0218

ORCID: Manfredo Atzori, PhD: 0000-0001-5397-2063; Claudio Castellini, PhD: 0000-0002-7346-2180; Barbara Caputo, PhD: 0000-0001-7169-0158; Anne-Gabrielle Mittaz Hager, PhD: 0000-0001-9461-2937; Simone Elsig: 0000-0003-2364-1671; Franco Bassetto, MD: 0000-0003-4105-8252; Henning Müller, PhD: 0000-00016800-9878

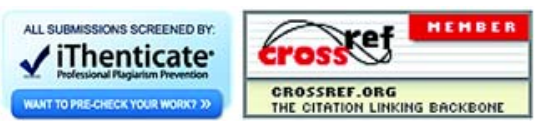

